\title{
Gait Recognition by Dynamic Cues
}

\author{
Imed Bouchrika \& Mark S. Nixon \\ Department of Electronics and Computer Science \\ University of Southampton, SO17 1BJ, UK \\ \{ib04r,msn\}@ecs.soton.ac.uk
}

\begin{abstract}
Many studies have now shown that it is possible to recognize people by the way they walk. As yet there has been little formal study of people recognition using the kinematic-related gait features. We present a new method for gait recognition using dynamic features including the angular measurements of the lower limbs as well as the spatial displacement of the trunk. Gait signatures are derived using Feature selection algorithm which is based on a validation-criterion. We show that gait angular measurements derived from the joint motions mainly the ankle, knee and hip angles, possess most of the discriminatory potency for gait recognition with an achieved correct classification rate of $95.7 \%$.
\end{abstract}

\section{Introduction}

The interest in gait as a biometric is strongly motivated by the need for an automated recognition system for visual surveillance and monitoring applications. Recently, the use of gait for people identification in surveillance applications has attracted researchers from computer vision. The suitability of gait recognition for surveillance systems emerges from the fact that gait can be perceived from a distance as well as its non-invasive nature. Although gait recognition is still a new biometric and is not sufficiently mature to be deployed in real world applications such as visual surveillance, it overcomes most of the limitations that other biometrics suffer from such as face, fingerprints and iris recognition which can be obscured in most situations where serious crimes are involved.

The gait features used for recognition can be broadly classified into two categories, namely static and dynamic cues. The static features reflect the geometrybased measurements of the anatomical structure of the human body such as the person's height and the length or width of the different body segments. The static fea- tures can also be derived from the observed gait such as the stride length. The dynamic features are the cues which describe the kinematics of the locomotion process, such as the angular motion of the lower limbs extracted from the joint trajectory data. As the static cues are less taxing to extract and compute, it would seem straightforward to recognise people using static features such as the stride and body height and so forth. Furthermore, recent research on gait using static features for identification proved that a promising recognition rate can be reached $[2,14]$.

On the other hand, some researchers have preferred to fuse both static and dynamic cues with a belief that fusion would yield the optimal gait recognition rate. Wagg [13] proposed a model-based method for recovering the joint angular motion and the static parameters of the human body such as the thigh width. The f-statistic scores are used as coefficients to weight the discriminative features. Although a high recognition rate was reported, it was clearly noted that the body-related parameters are not robust [13] since they are highly dependent on the clothing of walking subjects.

Despite the fact that static features were proved by recent experiments to achieve promising gait recognition rates, their use for the development of a biometric system is impractical. This is mainly because static features are dependent on clothing, bags, and other factors $[13,12]$ which would certainly affect the recognition performance. On the other hand, Cutting et al. [5] argued that dynamic features contribute significantly more in human recognition than static cues such as height. Cutting concluded that kinematic information is important for gender classification and static features are insufficient to reveal the gender of the walking subject. Moreover, Stevenage et al. [10] supported the argument that gait-related cues are more important than body-related features for the purpose of human identification. This conclusion was based on an experiment carried out on 6 different walkers where it was reported that shape information has no effect on the recogni- 
tion performance. The answers to the crucial questions of whether kinematic information offers better discriminability than static features, will be laid out in the following sections.

\section{Derivation of Gait Signature}

To extract the gait features of walking subjects from the covariate dataset, the model-based method proposed in [3] is employed to automate the extraction process of the joint trajectories. Motion templates describing the motion of the joints are derived by manual gait analysis. A recursive evidence gathering algorithm is emplopyed for the extraction process whereby spatial motion models are presented in a parameterized form using the Elliptic Fourier Descriptors described in equation (1):

$\left[\begin{array}{l}x(t) \\ y(t)\end{array}\right]=\left[\begin{array}{l}a_{0} \\ b_{0}\end{array}\right]+\left[\begin{array}{cc}\cos (\alpha) & -\sin (\alpha) \\ \sin (\alpha) & \cos (\alpha)\end{array}\right]\left[\begin{array}{c}F_{x}(t) * s_{x} \\ F_{y}(t) * s_{y}\end{array}\right]$

where $\alpha$ is the rotation angle, $s_{x}$ and $s_{y}$ are the scaling factors across the horizontal and vertical axes respectively. $a_{0}$ and $b_{0}$ define the position of the shape's centre. $F_{x}(t)$ and $F_{y}(t)$ are computed using equation:

$$
\begin{aligned}
& F_{x}(t)=\sum_{k=1}^{n} a_{x_{k}} \cos (k t)+b_{x_{k}} \sin (k t) \\
& F_{y}(t)=\sum_{k=1}^{n} a_{y_{k}} \cos (k t)+b_{y_{k}} \sin (k t)
\end{aligned}
$$

where $a_{x_{k}}, a_{y_{k}}, b_{x_{k}}$ and $b_{y_{k}}$ are the set of the elliptic phasors which can be computed by Riemann summation [1]. Gait knowledge is exploited via heel strike extraction to reduce the the parameter space dimensionality and therefore reduce the computational load of the evidence gathering algorithm.

The processing and derivation of potent gait features from this trajectory-based data, is still an unsolved and challenging problem [6] due to the complexity of the human visual perception system as well as the compound nature of gait motion inherent in the numerous variables associated with it including kinematics, kinetics and anthropometrics [4]. An important issue in gait recognition is the derivation of appropriate features that can capture the discriminative individuality from the subject's gait. Such features should respond to crucial criteria such as robustness and invariance to weather conditions, clothing and operating conditions.

In order to identify a subject by their gait, we derive the angular measurements as well as the trunk spatial displacment which best describe the gait kinematics. The use of angular motion is very common in gait analysis and recognition. The angles of the joints including the hip and the knee; are considered the most important kinematics of the lower limbs. Additional gait parameters are derived by fusing together the gait angles associated with the hip, knee and ankle. The gait vector is composed from the angle between the thighs as the sum of the two hip angles. The vector also includes additional measures produced by fusing the right and left angles of the knee as well as the ankle. Fusion of cues is achieved by applying simple rules including summation, product and difference.

\section{Gait Feature Selection}

Feature selection is an important task for almost any pattern recognition problem. This procedure is aimed to derive as many discriminative cues as possible whilst removing the redundant and irrelevant information which may degrade the recognition rate. It is practically infeasible to run an exhaustive search for all the possible combinations of features in order to obtain the optimal subset for recognition due to the high dimensionality of the feature space. For this reason, we employed the Adaptive Sequential Forward Floating Selection (ASFFS) search algorithm [9]

The feature selection procedure fundamentally relies on an evaluation function that determines the usefulness of each feature in order to derive the ideal subset of features for the classification phase. A number of methods [16] rely mainly on statistical metric measures which are based on the scatter or distribution of the training samples in the feature space such as the Bhattacharyya metric. Although, statistical methods enjoy low-cost implementation. they have been proved to offer poor estimate of the recognition rate because of their independence from the final classifier [15]. The algorithm uses a validation-based evalution criterion which is proposed to find the subset of features that minimises the classification errors as well as ensure good separability between the different classes. In contrast to the voting scheme used in the $K N N$, the evaluation function uses different weights $w$ to signify the importance of most nearest neighbours. The probability score for a sample $s_{c}$ to belong to class $c$ is expressed in the following equation (3):

$$
f\left(s_{c}\right)=\frac{\sum_{i=1}^{N_{c}-1} z_{i} w_{i}}{\sum_{i=1}^{N_{c}-1} w_{i}}
$$

where $N_{c}$ is the number of instances in class $c$, and the weight $w_{i}$ for the $i^{t h}$ nearest instance is inversely related to proximity as:

$$
w_{i}=\left(N_{c}-i\right)^{2}
$$


The value of $z_{i}$ is defined as:

$$
z_{i}=\left\{\begin{array}{cc}
1 & \text { if nearest }\left(s_{c}, i\right) \in c \\
0 & \text { otherwise }
\end{array}\right.
$$

such that the nearest $\left(s_{c}, i\right)$ function returns the $i^{\text {th }}$ nearest instance to the sample $s_{c}$. The Euclidean distance metric is employed to find the nearest neighbours. The subset significance based on the validation-based metric is estimated using the Leave-one-out crossvalidation rule.

\section{Experimental Results}

For the evaluation of dynamic gait features derived using the model-based method for people identification, a gallery dataset of 160 video sequences is taken from the SOTON indoor gait database. The set consists of 20 different subjects with 8 sequence for every individual.

\subsection{Classification Results}

Feature selection is applied on the video sequences to search for the most discriminative subset of features. Based on the validation criterion function, the feature selection algorithm derived 34,261 different feature subsets which achieved a recognition rate of $82 \%$ based on the probability scores explained in section (3). In order to further assess the recognition potency of the selected features, the Correct Classification Rate (CCR) is computed using the $K$-nearest neighbour (KNN) classifier with the Leave-one-out cross-validation rule. The $K N N$ rule is applied at the classification phase due to its low complexity and hence fast computation besides the ease of comparison to other methods.

A recognition rate of $95.7 \%$ is achieved for $k=5$ using the set of 160 video sequences . This is achieved using solely features describing purely the dynamics of the locomotion process. The results of the recognition performance are summarized in Table (1) with comparative results of other methods which use dynamic parameters for gait recognition. The correlation matrix is shown in Figure (4.1) which visualizes the separation values between the different classes. The darker squares reflect higher separation score and therefore higher discriminability. The white diagonal line reflects the zero distance between the same class. The separation value between the different classes is estimated using the Bhattacharyya distance metric.

To further assess the classification performance of the proposed method for gait recognition using dynamic-related features, a different probe dataset which had not been used for feature selection, is taken

\begin{tabular}{|l|l|l|}
\hline Method Name & Database & CCR \\
\hline Our method & 20 subjects, 160 sequences & $95.7 \%$ \\
\hline Bobick [11] & 18 subjects, 106 sequences & $73 \%$ \\
\hline Yam [16] & 20 subjects, 100 sequences & $84 \%$ \\
\hline Wang [14] & 20 subjects, 80 sequences & $87.5 \%$ \\
\hline
\end{tabular}

Table 1. Gait Recognition Results using Dynamic Cues

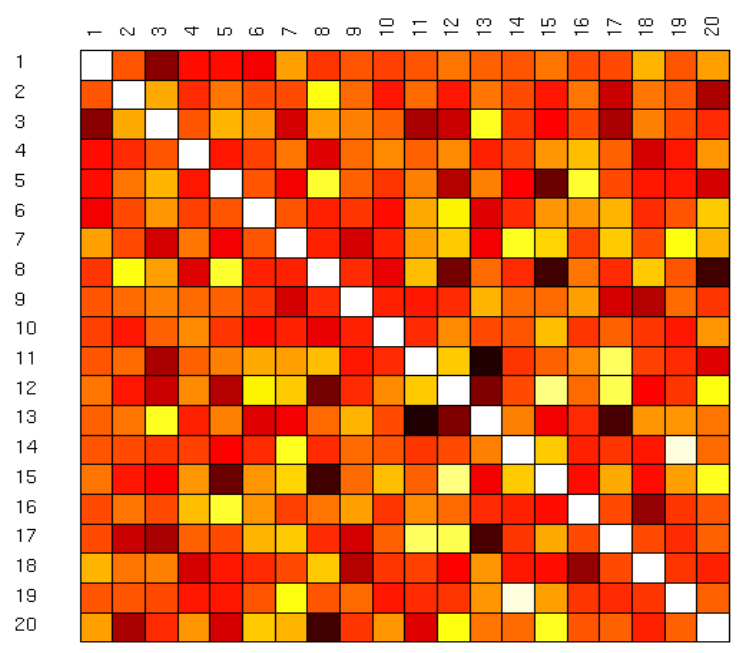

Figure 1. Correlation Matrix for Gait Recognition

from the SOTON gait database and matched against the gallery dataset. The dataset is composed of $60 \mathrm{se}-$ quences for 20 subjects with 3 sequences for every individual. Using the Cumulative Match Score (CMS) evaluation method which was introduced by Phillips $e t$ al in the FERET protocol [8], we have correctly classified $86.67 \%$ of the 60 walking sequences at rank $R=1$. The results achieved using this evaluation are promising because the probe set has not been employed for the derivation of the dynamic feature subset. Henceforth, the derived dynamic features have a potential discriminative capability to identify people.

\subsection{Gait Feature Analysis}

In order to determine what dynamic features are important for the identification of people, the different gait-related features are analysed separately to estimate their contribution and significance for gait recognition. To produce accurate and unbiased results, we have derived a number of 493 subsets using the validationbased criteria described in section (3) for feature analysis. The feature subsets which are of length between 22 
and 54, achieved a Correct Recognition Rate of $92.15 \%$ or over using the KNN classifier. The distributions and recognitio rates of the different types of features are illustrated in Table (2). The distribution values provide an indication whether such type of feature is important for the recognition process but it does not provide a measure of its discriminatory potency. Instead, the discriminative significance of dynamic features is approximated using the correct recognition rate.

\begin{tabular}{|l|c|c|}
\hline Features & Distribution & Recognition Rate \\
\hline Hip Angle & $27 \%$ & $45 \%$ \\
\hline Knee Angle & $29 \%$ & $52 \%$ \\
\hline Ankle Angle & $21 \%$ & $52.5 \%$ \\
\hline$X$ disp & $16 \%$ & $26 \%$ \\
\hline$Y$ disp & $7 \%$ & $25 \%$ \\
\hline
\end{tabular}

Table 2. Gait Recognition Results using Dynamic Cues

The angular measurements possess most of the discriminative features with an average proportion of $77 \%$ of the gait signature, whilst only a few features are embedded in the displacement motion. The gait angular features are derived from the knee, ankle and hip angles with proportions of $27 \%, 29 \%$ and $21 \%$ respectively. The knee and ankle angular features are observed to be the most discriminative features with an achieved recognition rate of $52 \%$ and $52.5 \%$ respectively. These analytical results are consistent with the medical reports in [7] whereby Murray observed that the ankle rotation, pelvic tipping and spatial displacements of the trunk embed the subject individuality due to their consistency at different trials. In [13], Wagg has recently confirmed the importance of the angular features for gait recognition with a reported recognition rate of $77 \%$ using dynamic gait features.

\section{Conclusions}

In this paper, experimental research is carried out to confirm the early psychological theories claiming that the discriminative features for motion perception and people recognition are embedded in gait kinematics. Dynamic versus static features are reviewed and discussed with their potentials for people identification using gait. We have shown that the gait angular measurements derived from the joint motions mainly the ankle, knee and hip angles, posses most of the discriminatory potency for gait recognition with an achieved correct classification rate of $95.7 \%$.

\section{References}

[1] A. S. V. Aguado, M. S. Nixon, and M. E. Montiel. Parameterizing Arbitrary Shapes via Fourier Descriptors for Evidence-Gathering Extraction. Computer Vision and Image Understanding, 69(2):202-221, 1998.

[2] A. F. Bobick and A. Johnson. Gait Recognition using Static Activity-Specific Parameters. In Proceedings of the IEEE Conference on Computer Vision and Pattern Recognition, 1:423-430, 2001.

[3] I. Bouchrika and M. S. Nixon. Model-Based Feature Extraction for Gait Analysis and Recognition. In Proceedings of Mirage: Computer Vision / Computer Graphics Collaboration Techniques and Applications, pages 150-160, 2007.

[4] T. Chau. A review of analytical techniques for gait data. Part 1: fuzzy, statistical and fractal methods. Gait Posture, 13(1):49-66, 2001.

[5] J. E. Cutting and D. Proffitt. Gait Perception as an Example of How we may Perceive Events. Intersensory perception and sensory integration, pages 249273, 1981.

[6] L. Lee and W. Grimson. Gait Analysis for Recognition and Classification. In Proceedings of Fifth IEEE International Conference on Automatic Face and Gesture Recognition., pages 148-155, 2002.

[7] M. P. Murray. Gait as a Total Pattern of Movement. American Journal of Physical Medicine, 46(1):290333, 1967.

[8] P. J. Phillips, H. Moon, S. A. Rizvi, and P. J. Rauss. The FERET Evaluation Methodology for Face Recognition Algorithms. IEEE Transactions on Pattern Analysis and Machine Intelligence, 22(10):1090-1104, 2000.

[9] P. Somol, P. Pudil, J. Novovičová, and P. Paclík. Adaptive Floating Search Methods in Feature Selection. Pattern Recognition Letters, 20(11-13):1157-1163, 1999.

[10] S. V. Stevenage, M. S. Nixon, and K. Vince. Visual Analysis of Gait as a Cue to Identity. Applied Cognitive Psychology, 13(6):513-526, 1999.

[11] R. Tanawongsuwan and A. Bobick. Gait recognition from time-normalized joint-angle trajectories in the walking plane. IEEE Conf on Computer Vision and Pattern Recognition, pages 726-731, 2001.

[12] G. V. Veres, M. S. Nixon, and J. N. Carter. Modelling the Time-Variant Covariates for Gait Recognition. in Proc of 5th Intl conf on Audio-and Video-Based Viometric Person Authentication, pages 597-606, 2005.

[13] D. K. Wagg and M. S. Nixon. On Automated ModelBased Extraction and Analysis of Gait. Conf Automatic Face and Gesture Recognition, pages 11-16, 2004.

[14] L. Wang, H. Ning, T. Tan, and W. Hu. Fusion of Static and Dynamic Body Biometrics for Gait Recognition. IEEE Transactions on Circuits and Systems for Video Technology, 14(2):149-158, 2004.

[15] A. R. Webb. Statistical Pattern Recognition. Hodder Arnold, 1999.

[16] C. Y. Yam, M. S. Nixon, and J. N. Carter. Automated Person Recognition by Walking and Running via Model-Based Approaches. Pattern Recognition, 37(5):1057-1072, 2004. 